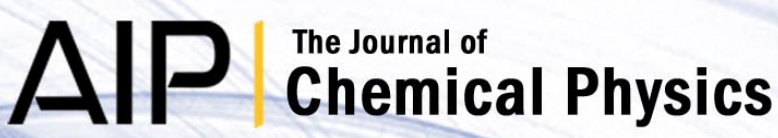

The $\mathrm{D}+\mathrm{H} 2(\mathrm{v}=1, \mathrm{j}) \rightarrow \mathrm{HD}\left(\mathrm{v}^{\prime}, \mathrm{j}^{\prime}\right)+\mathrm{H}$ reaction. A detailed quasiclassical trajectory study

F. J. Aoiz, H. K. Buchenau, V. J. Herrero, and V. Sáez Rábanos

Citation: J. Chem. Phys. 100, 2789 (1994); doi: 10.1063/1.466473

View online: http://dx.doi.org/10.1063/1.466473

View Table of Contents: http://jcp.aip.org/resource/1/JCPSA6/v100/i4

Published by the American Institute of Physics.

\section{Additional information on J. Chem. Phys.}

Journal Homepage: http://jcp.aip.org/

Journal Information: http://jcp.aip.org/about/about_the_journal

Top downloads: http://jcp.aip.org/features/most_downloaded

Information for Authors: http://jcp.aip.org/authors

\section{ADVERTISEMENT}

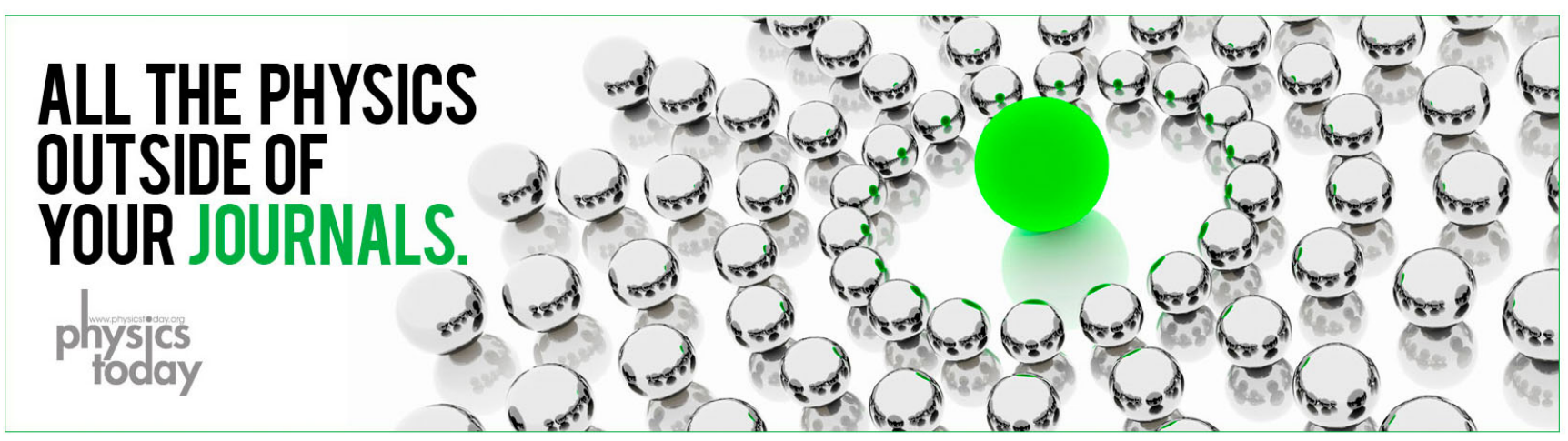




\title{
The $D+H_{2}(v=1, j) \rightarrow H D\left(v^{\prime}, j^{\prime}\right)+H$ reaction. A detailed quasiclassical trajectory study
}

\author{
F. J. Aoiz \\ Departamento de Química Física I, Facultad de Química, Universidad Complutense, 28040 \\ Madrid, Spain \\ H. K. Buchenaua) \\ Max-Planck Institut für Strömungsforschung, Bunsenstrasse 10, 3400 Göttingen, Germany \\ V. J. Herrero \\ Instituto de Estructura de la Materia (CSIC), Serrano 123, 28006 Madrid, Spain \\ V. Sáez Rábanos \\ Departamento de Quimica General y Bioquímica, ETS Ingenieros de Montes, Universidad Politécnica, \\ 28040 Madrid, Spain
}

(Received 23 August 1993; accepted 1 November 1993)

Thorough quasiclassical trajectory (QCT) calculations have been carried out for the $\mathbf{D}+\mathrm{H}_{2}(v$ $=1, j$ ) exchange reaction. These calculations include integral and differential cross sections, rate constants, reaction probabilities as a function of total energy, opacity functions, and distributions of internal states of the HD product in the range of collision energies from the reaction threshold to $1.5 \mathrm{eV}$ and initial $j$ values from 0 to 12 . An overall good agreement with some discrepancies is found between the present QCT results and those from experiments and accurate quantum-mechanical calculations.

\section{INTRODUCTION}

The reaction $\mathrm{D}+\mathrm{H}_{2}(v=1) \rightarrow \mathrm{HD}+\mathrm{H}$ has been extensively studied over a long period of time. ${ }^{1}$ It is one of the isotopic variants of the prototypic $\mathrm{H}_{3}$ system, for which the potential energy surface (PES) is known with great accuracy $^{2-5}$ and offers the best perspectives for a detailed study of vibrational excitation in the dynamics of an elementary reaction.

Rate constants for $\mathrm{D}+\mathrm{H}_{2}(v=1)$ have been measured since the 1970 s by a variety of techniques. ${ }^{6-13}$ The comparison of the first experimental rate constants with theoretical results, which at the time had been obtained from quasiclassical trajectory (QCT) calculations ${ }^{14,15}$ as well as from several quantum-mechanical (QM) approximate methods ${ }^{16-25}$ showed in most cases a surprising disagreement, the theoretical rate constants being (in general) much smaller (in the case of QCT nearly an order of magnitude) than the experimental ones. This controversy stimulated both new measurements and calculations. In fact, an exact QM calculation by Truhlar and co-workers ${ }^{26}$ for total angular momentum $J=0$ gave a threshold value in agreement with previous theoretical treatments, too high to be compatible with the existing experimental rate constants. In experiments using coherent anti-Stokes Raman spectroscopy (CARS) for the detection of both, the $\mathrm{H}_{2}(v$ $=1$ ) reactant and the $\mathrm{HD}$ product, Dreier and Wolfrum ${ }^{27}$ obtained a $k$ value $\left(k=1.0 \pm 0.4 \times 10^{-13} \mathrm{~cm}^{3} \mathrm{~s}^{-1}\right)$ in reasonable agreement with theoretical predictions. An ulterior refinement of this experiment ${ }^{1,28}$ allowed the direct measurement of the hydrogen atom concentration and the determination of the rate constants for the production of HD

a) Present address: JILA, University of Colorado, Boulder CO 80309-440. in a specific vibrational state: $k=(1.7 \pm 0.5) \times 10^{-13}$ $\mathrm{cm}^{3} \mathrm{~s}^{-1}$ for $\mathrm{HD}\left(v^{\prime}=1\right)$ and $k=(4.3 \pm 1.3) \times 10^{-14}$ $\mathrm{cm}^{3} \mathrm{~s}^{-1}$ for $\mathrm{HD}\left(v^{\prime}=0\right)$ at $330 \mathrm{~K}$. These measurements improved the agreement with $\mathrm{QCT}^{15}$ and with most QM approximate calculations and were corroborated by a basically exact $\mathrm{QM}$ calculation carried out by Zhang and Miller $^{29}$ for $\mathrm{D}+\mathrm{H}_{2}(v=1, j=0) \rightarrow \mathrm{HD}+\mathrm{H}$ on the LiuSiegbahn-Truhlar-Horowitz (LSTH) ${ }^{2}$ PES by using a method based on the Kohn variational principle.

A more direct test of theoretical predictions is provided by experiments with microscopic resolution (i.e., yielding cross sections instead of rate constants). A crossed molecular beam arrangement was used by Toennies and co-workers ${ }^{30-33}$ for the investigation of the $\mathrm{D}+\mathrm{H}_{2}(v=0,1)$ reaction. The experiment was initially performed at an average collision energy $\bar{E}_{T}=0.33 \mathrm{eV}$,- not far from the threshold for the reaction of $\mathrm{H}_{2}$ excited to $v=1$. The $\mathrm{D}$ atoms were generated in a $D_{2}$ microwave discharge and the internal excitation of the $\mathrm{H}_{2}$ molecules was achieved by expanding hydrogen from a tungsten nozzle inductively heated to $2800 \mathrm{~K}$. This led to a $\sim 10 \%$ population in the $v=1$ state of $\mathrm{H}_{2}$ but also to a broad distribution of rotational states. Due to the width of the collision energy distribution ( $F W H M \sim 0.15 \mathrm{eV}$ ) the contribution from the $\mathrm{D}+\mathrm{H}_{2}(v=0)$ reaction was significant. Angular and timeof-flight (TOF) distributions of the HD molecules were measured with a movable electron bombardment mass spectrometer. A calibration of the apparatus with $\mathrm{D}_{2}+\mathrm{H}_{2}$ elastic scattering made possible the determination of the absolute integral cross section ${ }^{31,32}$ for the process $\mathbf{D}+\mathrm{H}_{2}(v$ $=1, j=0) \rightarrow \mathrm{HD}+\mathrm{H}$ at the collision energy $E_{T}=0.33 \mathrm{eV}$. The estimated value of this cross section was $\sigma_{R}=1.14$ $\pm 0.34 \dot{\mathrm{A}}^{2}$. The theoretical differential cross sections (DCS) existing at the time [from QCT and reactive infinite 
order sudden approximation (RIOSA) were taken as the basis for the simulation of the measured distributions. In order to get good concordance it was necessary to take into account the marked nonmonotonic dependence of the cross section $\sigma_{R}$ on the rotational quantum number $j$ of $\mathrm{H}_{2}(v=1)$ at $E_{T}=0.33 \mathrm{eV}$ obtained by Sathyamurthy and Toennies $^{34}$ in a QCT calculation. Subsequent trajectory calculations by Aoiz et al. ${ }^{35,36}$ corroborated this effect for a wider range of collision energies and for $v=0,1$, and 2 . Accurate quantum-mechanical calculations for $\mathrm{D}+\mathrm{H}_{2}(v$ $=1) \rightarrow \mathrm{HD}+\mathrm{H}$ have been performed by Zhang and Miller $^{29}$ for collision energies similar to those of the experiment; they are, however, limited to $j=0$ and cannot be directly compared to the measurements which include large $j$. The differential cross sections obtained in QCT calculations for $j=0$ and used for the simulation of the experiment are in good agreement with the corresponding $\mathrm{QM}$ ones.

The experiment just described was refined further ${ }^{33}$ by decreasing the spread of the collision energy distribution and by extending the measurements to lower $E_{T}$ values $\left(E_{T}=0.30,0.28\right.$, and $\left.0.24 \mathrm{eV}\right)$ closer to the threshold for reaction of $\mathrm{H}_{2}$ in the $v=1$ level and with a much smaller contribution from the ground-state channel. In spite of the increased resolution it was not possible to clearly separate the final vibrational states of HD in the measured distributions. The simulation of the laboratory results was also improved by inlcuding QCT state resolved differential cross sections for a finer grid of collision energies and initial $j$ values. Part of the results of the detailed calculations needed for this comparison are reported in the present work.

A different experimental approach to the study of this reaction has been followed by the group of Zare ${ }^{37-40}$ by using a combination of laser techniques. These experiments have enabled the measurement of state-to-state integral cross sections and rate constants for $\mathrm{D}+\mathrm{H}_{2}(v=1$, $j=1) \rightarrow \mathrm{HD}\left(v^{\prime}=0,1,2 ; j^{\prime}\right)+\mathrm{H}$ at higher collision energies $\left(E_{T} \sim 0.8-1.5 \mathrm{eV}\right)$ than those sampled in the experiments mentioned above. Fast $\mathrm{D}$ atoms were generated by laser photolysis of either DBr or DI and the excitation of the $\mathrm{H}_{2}$ molecule to the $v=1, j=1$ state was achieved by means of stimulated Raman pumping. The $\operatorname{HD}\left(v^{\prime}, j^{\prime}\right)$ product was detected through $(2+1)$ resonance-enhanced multiphoton ionization (REMPI). In the first version of this experiment, ${ }^{37,38}$ a mixture of $\mathrm{DBr}$ and $\mathrm{H}_{2}$ flowed into the vacuum chamber; where the same laser generated the $\mathrm{D}$ atoms and ionized the HD molecules. The experimentally deduced integral cross sections for the process $\mathrm{D}+\mathrm{H}_{2}(v=1$, $j=1) \rightarrow \mathrm{HD}\left(v^{\prime}=1, j^{\prime}\right)+\mathrm{H}$ at $E_{T}=1.03 \mathrm{eV}$ were compared to the results of converged quantum-mechanical calculations by Blais $e t a l^{41}$ and by Mielke et $a l^{42}$ carried out on the double many-body expansion (DMBE) ${ }^{3}$ surface by using a method based on the generalized Newton variational principle (GNVP). In addition, Blais et al. ${ }^{41}$ performed QCT calculations for the same system. The experimental $j^{\prime}$ distribution was broad, extending at least to $j^{\prime}=12$ and with a sharp peak at $j^{\prime}=7$. The theoretical calculations (from both QCT and QM methods) also yielded broad distributions but more asymmetrical and peaking at about $j^{\prime}=10$. The shapes of the two theoretical distributions were quite similar, but at the maximum, the QM integral cross section was larger (by about $25 \%$ ) than the QCT one. Later, new calculations were carried out on three different $\mathrm{PES}^{2-5}$ by Keogh et al. ${ }^{43}$ Although they specifically took into account the contribution to the reaction of $D$ atoms with lower translational energies also coming from the photolysis of $\mathrm{DBr}$, their results basically corroborated the previous ones and thus the disagreement between experiment and theory. Detailed experimental checks ${ }^{39}$ have indicated that space-charge effects associated with the use of $\mathrm{DBr}$ as the photolytic precursor of the $\mathrm{D}$ atoms may interfere with the detection of the higher $j^{\prime}$ states of the HD product, which is the region of the experimental $j^{\prime}$ distribution at variance with the calculations. The experiment was further improved by substituting the $\mathrm{DBr}$ precursor with DI and by introducing an arrangement in which the photolysis laser was independent of the probe laser. The state specific rate constants measured ${ }^{39,40}$ were compared to those obtained from a detailed quantummechanical calculation carried out by Neuhauser and coworkers ${ }^{40}$ by using a newly developed time-dependent wave packet approach. There is an overall good agreement between the experimental and theoretical rotationally resolved rate constants, but for the highest collision energies studied $\left(E_{T}=1.4,1.5 \mathrm{eV}\right)$ the theoretical distributions peak one or two quanta higher than the experimental ones. Very recent accurate $\mathrm{QM}$ calculations by $\mathrm{Wu}$ and Kuppermann $^{44,45}$ using a symmetrized hyperspherical coordinate formalism indicate that taking into account the effect of the geometric phase induced by the conical intersection between the two lowest potential energy surfaces of the $\mathrm{H}+\mathrm{H}_{2}$ system can lead to an excellent agreement with all the measured rotational distributions (including the one from the $\mathrm{DBr}$ experiment).

In view of the rich information available on the dynamics of $\mathrm{D}+\mathrm{H}_{2}(v=1) \rightarrow \mathrm{HD}+\mathrm{H}$ we have attempted a comprehensive QCT study of this reaction. In the present work we report the results of this study. These calculations have been carried out on the LSTH potential energy surface and include thermal rate constants, integral and differential reaction cross sections, reaction probabilities as a function of total energy and of total angular momentum, and distributions of internal states of the HD product in the range of collision energies from threshold to $1.5 \mathrm{eV}$. The flexibility of the QCT method has made possible the consideration of the very different initial conditions needed for the simulation of the various experiments (i.e., large rotational quantum number for the molecular beam measurements, high collision energies for the laser ones). The results of the calculations are discussed and compared to the experimental data and to other theoretical treatments.

\section{METHOD}

The general method used in the QCT calculation has been described elsewhere (see Refs. 36 and 46, and references cited therein) and only the details relevant to this work will be given here. 
All the trajectories have been calculated on the LSTH PES with $\mathrm{H}_{2}$ in $v=1$. The total number of trajectories used in this work has been $1.45 \times 10^{6}$. Of these $4 \times 10^{5}$ correspond to $v=1, j=0$ and $3 \times 10^{5}$ to $v=1, j=1$.

Integration algorithms and tests of accuracy are as in Ref. 46. A conservation of 1 in $10^{5}$ and 1 in $10^{7}$ in total energy and angular momentum, respectively, is achieved with a step size of $5 \times 10^{-17} \mathrm{~s}$. For $j=0$ the collision energy is swept from 0.25 to $1.00 \mathrm{eV}$ in intervals of $0.05-0.10$ $\mathrm{eV}$, and a finer grid is used in between 0.13 and $0.25 \mathrm{eV}$ to accurately determine the classical threshold. Similar resolution in energy is used for $j=1-4$.

Calculation of final $v^{\prime}, j^{\prime}$ quantum numbers is done, as in previous works, by equating the internal energy to the full Dunham expansion of vibrorotational energies of the HD molecule, subject to the constraint of a given rotational angular momentum of $\mathrm{HD}$. The values of $v^{\prime}, j^{\prime}$ thus found are then simply rounded to the nearest integer. No other assignment scheme was tried in this work.

To determine the reaction probability as a function of total energy for $v=1, j=0$ additional $4 \times 10^{4}$ trajectories were calculated with the impact parameter $b=0$ (hence total angular momentum $J=0$ ). The collision energy was randomly sampled in a uniform way in the interval 0.15 to $1.00 \mathrm{eV}$. The dependence on the total energy of the reaction probability $P(E ; J=0)$ was then fitted to a Legendre polynomial series as indicated in Ref. 46 . To further check the accuracy of this fit two more batches of 6000 trajectories each were run at $b=0$ at the single collision energies of $E_{T}=0.70$ and $0.90 \mathrm{eV}$, respectively.

The translational excitation functions for $v=1, j=0$ to 4 were fitted by weighted least squares to suitable functions which then were used to evaluate the thermal rate constants $k(T ; v=1, j)$. These results were further averaged on initial $j(j=0,1,2,3$, and 4$)$ to yield $k(T ; v=1)$. The statistical uncertainties of these $k(T)$ are determined by standard error propagation using the full covariance matrix ${ }^{47}$ of the fits which include the uncertainties of individual values of $\sigma_{R}\left(E_{T}\right)$.

In order to simulate the laser experiments reported in Ref. 39 with present QCT calculations, the state specific rate constant including the contributions of the fast and slow D atom channels arising from the photolysis of DI (see below) is written as

$$
k\left(v=1, j=1 \rightarrow v^{\prime}, j^{\prime}\right)=\sigma_{R}\left(E_{T}^{s}\right) v_{r}^{s} p_{\mathrm{D}}^{s}+\sigma_{R}\left(E_{T}^{f}\right) v_{r}^{f} p_{\mathrm{D}}^{f},
$$

where the superscripts $s$ and $f$ make reference to the slow and fast $\mathrm{D}$ atom channels, $\sigma_{R}\left(E_{T}\right)$ are the specific $v^{\prime}, j^{\prime}$ integral cross sections, $v_{r}$ are the relative velocities, and $p$ are the fractions of $\mathrm{D}$ atoms produced in each channel such that $p_{\mathrm{D}}^{f}+p_{\mathrm{D}}^{s}=1$.

Following Ref. 39 and for the simulation carried out here, the $p_{\mathrm{D}}$ fractions of each channel for the following nominal energies are taken to be $p_{\mathrm{D}}^{f}=0.74, p_{\mathrm{D}}^{s}=0.26$ for $E_{T}=0.80 \mathrm{eV} ; p_{\mathrm{D}}^{f}=0.54, p_{\mathrm{D}}^{s}=0.46$ for $E_{T}=1.30 \mathrm{eV} ; p_{\mathrm{D}}^{f}$ $=0.62, p_{\mathrm{D}}^{s}=0.38$ for $E_{T}=1.40 \mathrm{eV}$ and finally, $p_{\mathrm{D}}^{f}=0.77$, $p_{\mathrm{D}}^{s} \equiv 0.23$ for $E_{T}=1.50 \mathrm{eV}$. In fact each one of the experiments at $E_{T}=1.30,1.40$, and $1.50 \mathrm{eV}$ involves a range of
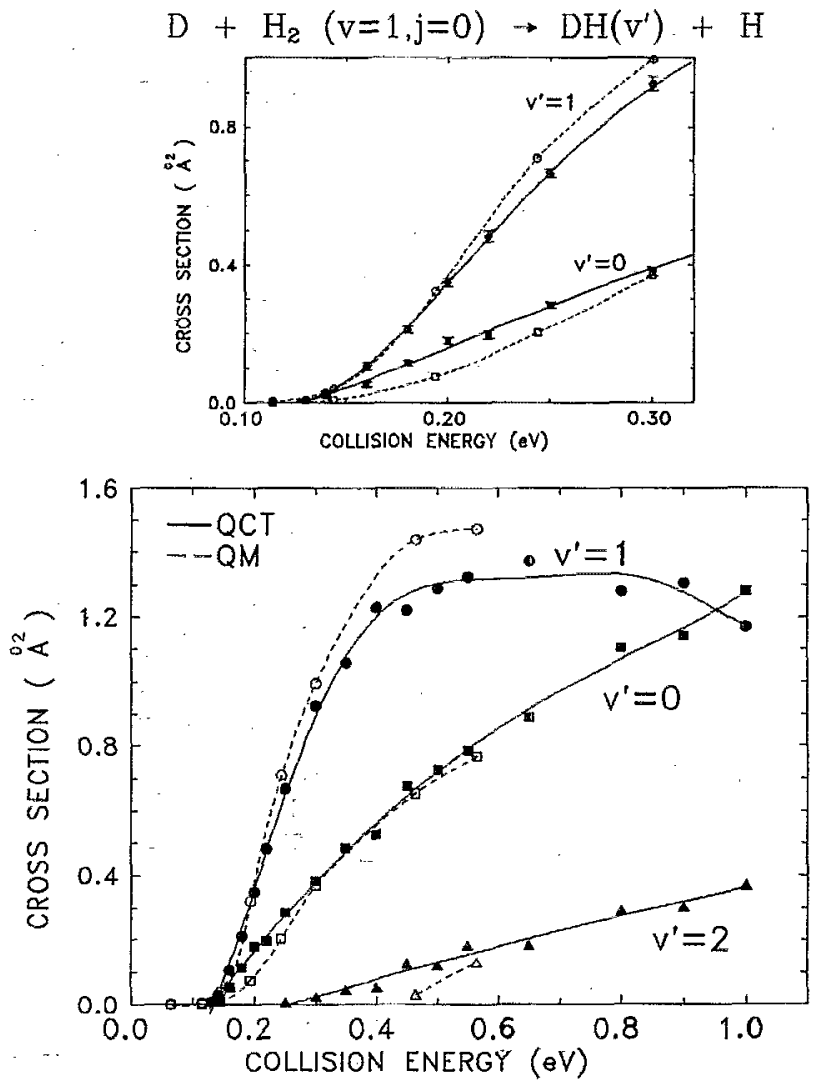

FIG. 1. Vibrationally state resolved reaction cross section $\left(\AA^{2}\right)$ as a function of the translational energy for the $\mathrm{D}+\mathrm{H}_{2}(\dot{v}=1, j=0)$ reaction. Filled symbols-solid lines are present $\mathrm{QCT}$ results, open symbolsdashed lines are accurate QM values from Ref. 29; both sets of data calculated on the LSTH PES (Ref. 2). Squares are for $v^{\prime}=0$, circles for $v^{\prime}=1$, and triangles for $v^{\prime}=2$. The upper part of the figure is a detailed view of the threshold region. Where not shown, QCT error bars (one standard deviation) are smaller than the points.

$p_{\mathrm{D}}^{f}$ and $p_{\mathrm{D}}^{s} ;$ those taken here are intermediate values selected within this range. The experimental results are then scaled by means of a weighted least-squares fit to the theoretical ones (in units of $\mathrm{cm}^{3} \mathrm{~s}^{-1}$ ) taking into account both the uncertainties in the experimental and in the QCT specific rate constants. ${ }^{47}$ It should be noticed that the experiments involve different collision energies depending on the final $v^{\prime}, j^{\prime}$ state detected and therefore a more accurate simulation would imply the calculation of the cross sections in a range of collision energies around the nominal one.

As in previous works ${ }^{36,46}$ the solid angle differential cross sections as well as the reaction probabilities as a function of the total angular momentum and their statistical uncertainties are calculated by the method of moments expansion in Legendre polynomials.

\section{RESULTS AND DISCUSSION}

The energy evolution of the integral reaction cross section for $\mathrm{D}+\mathrm{H}_{2}(v=1, j=0) \rightarrow \mathrm{HD}\left(v^{\prime}\right)+\mathrm{H}$ resolved into the final vibrational states of HD is shown in Fig. 1. For collision energies up to $0.9 \mathrm{eV}$ the vibrationally adiabatic 
TABLE I. Specific thermal rate constants $k\left(T ; v=1, j=0 \rightarrow v^{\prime}\right)\left(\mathrm{cm}^{3} \mathrm{~s}^{-1}\right)$ summed on final rotational states for the $\mathrm{D}+\mathrm{H}_{2}(v=1, j=0) \rightarrow \mathrm{HD}\left(v^{\prime}\right)+\mathrm{H}$ reaction.

\begin{tabular}{|c|c|c|c|c|c|c|c|}
\hline \multirow[b]{2}{*}{$T(\mathrm{~K})$} & \multicolumn{2}{|l|}{$\Sigma v^{\prime}$} & \multicolumn{3}{|c|}{$v^{\prime}=0$} & \multicolumn{2}{|l|}{$v^{\prime}=1$} \\
\hline & $\mathrm{QCT}^{\mathrm{a}}$ & $\mathrm{QM}^{\mathrm{b}}$ & & $\mathrm{QCT}^{2}$ & $\mathrm{QM}^{\mathrm{b}}$ & $\mathrm{QCT}^{\mathrm{n}}$ & $\mathrm{QM}^{\mathrm{b}}$ \\
\hline 200 & $(1.16 \pm 0.22) E-14$ & $1.12 \mathrm{E}-14$ & $(4.1 \pm 1$ & 1.0) $E-15$ & $2.31 E-15$ & $(7.6 \pm 1.0) \mathrm{E}-15$ & $8.88 \mathrm{E}-15$ \\
\hline 300 & $(2.03 \pm 0.20) E-13$ & $1.63 \mathrm{E}-13$ & $(6.77 \pm$ & $=0.82) \mathrm{E}-14$ & $3.33 E-14$ & $(1.35 \pm 0.10) E-13$ & $1.29 \mathrm{E}-13$ \\
\hline 400 & $(9.32 \pm 0.57) \mathrm{E}-13$ & $7.87 E-13$ & $(3.02 \pm$ & $=0.22) \mathrm{E}-13$ & $1.74 \mathrm{E}-13$ & $(6.28 \pm 0.30) \mathrm{E}-13$ & $6.12 \mathrm{E}-13$ \\
\hline 600 & $(4.82 \pm 0.17) \mathrm{E}-12$ & $4.70 \mathrm{E}-12$ & $(1.51 \pm$ & $=0.04) E-12$ & $1.16 \mathrm{E}-12$ & $(3.25 \pm 0.09) \mathrm{E}-12$ & $3.53 E-12$ \\
\hline 1000 & $(2.11 \pm 0.06) \mathrm{E}-11$ & $2.09 \mathrm{E}-11$ & $(6.62 \pm$ & $=0.22) \mathrm{E}-12$ & $5.56 \mathrm{E}-12$ & $(1.39 \pm 0.03) \mathrm{E}-11$ & $1.52 \mathrm{E}-11$ \\
\hline
\end{tabular}

Present work.

${ }^{b}$ Taken from Ref. 29.

channel is the preponderant one. The cross section for the production of $\operatorname{HD}\left(v^{\prime}=1\right)$ rises steeply from $E_{T}=0.128 \mathrm{eV}$ (the extrapolated energy threshold) to $E_{T} \simeq 0.40 \mathrm{eV}$ where it levels off. For collision energies higher than $0.9 \mathrm{eV}$ this cross section decreases and at $E_{T}=1 \mathrm{eV}$ is slightly smaller than the one for the production of HD in the ground vibrational state. The cross sections for the generation of $\mathrm{HD}\left(v^{\prime}=0\right)$ and $\mathrm{HD}\left(v^{\prime}=2\right)$ grow monotonically over the whole $E_{T}$ range considered. Also in Fig. 1 the QCT cross sections are compared to the results of the accurate QM calculations of Zhang and Miller ${ }^{29}$ on the same potential energy surface for values of the collision energy from threshold to $0.56 \mathrm{eV}$. The overall trend in the evolution of the cross sections to the specific $v^{\prime}$ states is very similar. The $\mathrm{QM}$ cross section to $\mathrm{HD}\left(v^{\prime}=1\right)$ is also the largest one and the one that rises more steeply with $E_{T}$, besides, it also levels off for collision energies higher than about $0.40 \mathrm{eV}$. However, there are quantitative discrepancies between the two theoretical approaches: The QM cross sections for $v^{\prime}=1$ are very similar for $E_{T} \leqslant 0.25 \mathrm{eV}$ but larger by about $10 \%$ than the QCT ones for values of $E_{T}$ between 0.4 and $0.6 \mathrm{eV}$. The QM threshold for the production of $\mathrm{HD}\left(v^{\prime}=2\right)$ is higher than the quasiclassical one due to the fact that trajectories ending with less energy than that corresponding to the $v^{\prime}=2$ state are counted as belonging to this level by the "boxing" procedure used to assign the quantum states. Also, in the upper part of Fig. 1, the lowenergy region is represented to compare in more detail with QM results. It is interesting to notice the very good agreement for $v^{\prime}=1$ at energies $<0.25 \mathrm{eV}$ although classically no reaction appears for $E_{T}<0.128 \mathrm{eV}$ and in the QM calculations reaction is possible for $E_{T}<0.06 \mathrm{eV}$. These cross sections are too small to have any important contribution to the rate constants for temperatures as small as $200 \mathrm{~K}$ (see below). In this low-energy interval the QCT $v^{\prime}=0$ cross section is slightly (but systematically) higher than the QM one. Overall the total reaction cross sections summed on $v^{\prime}$ from QCT and QM calculations on the same potential surface compare extremely well especially for energies below $0.5 \mathrm{eV}$ (see Ref. 36).

Once the translational excitation functions are known with enough accuracy the thermal rate constant can be readily determined. The QCT thermal rate constants for $\mathrm{D}+\mathrm{H}_{2}(v=1, j=0)$ obtained in the present work are compared in Table I with the results of the accurate QM cal- culations by Zhang and Miller. ${ }^{29}$ The concordance between the classical and quantum calculations is quite good for the reaction summed over the final states. The QCT are somewhat larger and the major discrepancies $(<20 \%)$ are found at $300-400 \mathrm{~K}$. At temperatures higher than $600 \mathrm{~K}$ the agreement is very good. These results can be explained by taking into account the $\sigma_{R}\left(E_{T}\right)$ behavior. Although the classical threshold for reaction $(0.128 \mathrm{eV})$ is higher than the QM one, the QCT $\sigma_{R}\left(E_{T}\right)$ increases with $E_{T}$ more steeply than the QM one. Even at $200 \mathrm{~K}$ the last effect is dominant and the classical $k(T)$ is larger. At temperatures high enough, the contribution of higher energies where $\mathrm{QM} \sigma_{R}\left(E_{T}\right)$ is larger than the classical one serves to balance the situation and improves the agreement.

Resolving the rate constants into the final vibrational states of the products the best coincidence is found for the adiabatic $v^{\prime}=1$ channel at the lower temperatures $(T$ $<400 \mathrm{~K}$ ) and for the $v^{\prime}=0$ channel at the higher ones. The largest discrepancies (up to 50\%) between QM and QCT results are found for the reaction into $v^{\prime}=0$ at $T<400 \mathrm{~K}$. Inspection of the upper part of Fig. 1 provides the explanation of these facts. At low $E_{T} \mathrm{QM}$ and classical $\sigma_{R}\left(E_{T}\right)$ are almost the same for reaction into $v^{\prime}=1$, whereas for $v^{\prime}=0$ classical cross sections are overestimated in the low range of energies with respect to the $\mathrm{QM}$ ones.

The present $k(T ; v=1)$ summed on $v^{\prime}$ are similar (within 15\%) to the ones obtained by Mayne and Toennies ${ }^{15}$ in a previous QCT calculation on the same PES at $300 \mathrm{~K}$, but their value is about one-half of ours (and of the accurate $\mathrm{QM}$ one) at $600 \mathrm{~K}$. The most recent experimental rate constants ${ }^{1}$ reported for this reaction are listed in Table II together with the corresponding QCT values obtained

TABLE II. Thermal rate constants $\left(\mathrm{cm}^{3} \mathrm{~s}^{-1}\right)$ averaged on thermal initial rotational states at $T=330 \mathrm{~K}$ for the $\mathrm{D}+\mathrm{H}_{2}(v=1) \rightarrow \mathrm{HD}\left(v^{\prime}\right)+\mathrm{H}$ reaction.

\begin{tabular}{cccc}
\hline & $k(T ; v=1)$ & $k\left(T ; v=1 \rightarrow v^{\prime}=0\right)$ & $k\left(T ; v=1 \rightarrow v^{\prime}=1\right)$ \\
\hline $\begin{array}{c}\text { Present } \\
\text { QCT }\end{array}$ & $(2.03 \pm 0.16) \mathrm{E}-13$ & $(6.6 \pm 1.1) \mathrm{E}-14$ & $(1.39 \pm 0.12) \mathrm{E}-13$ \\
Expt. $^{\mathrm{a}}$ & $2.1 \mathrm{E}-13^{\mathrm{b}}$ & $(4.3 \pm 1.3) \mathrm{E}-14^{\mathrm{a}}$ & $(1.7 \pm 0.5) \mathrm{E}-13^{\mathrm{a}}$ \\
\hline \hline
\end{tabular}

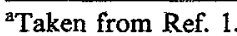

b Obtained by summing the $v^{\prime}=0$ and $v^{\prime}=1 k(T)$ reported in Ref. 1 . 


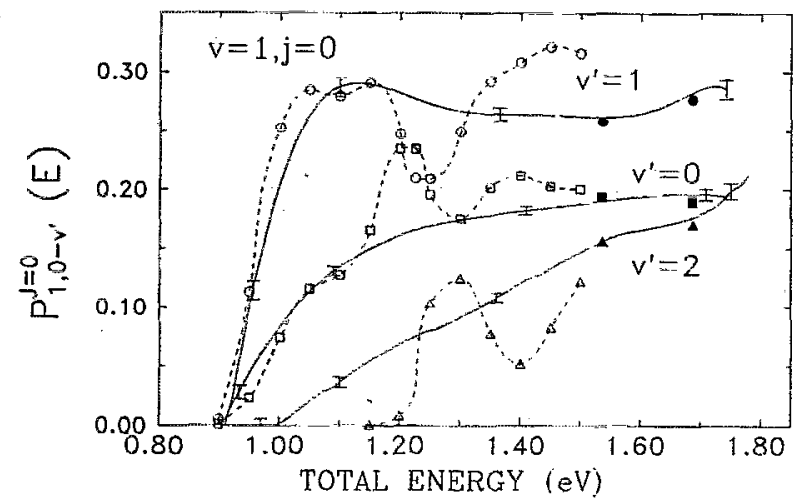

FIG. 2. Reaction probability at zero total angular momentum, $J=0$, into the final vibrational states $v^{\prime}=0,1,2$, summed on final rotational $j^{\prime}$ states for the $\mathrm{D}+\mathrm{H}_{2}(v=1, j=0)$ exchange reaction. The solid line with error bars are the QCT results of this work. Open symbols (squares $v^{\prime}=0$, circles $v^{\prime}=1$, triangles $v^{\prime}=2$ ) and the dashed line are the QM results of Zhang and Miller (Ref. 29). The solid points at 1.536 and $1.686 \mathrm{eV}$ represent the values obtained from the calculation at fixed collison energy and zero impact parameter in order to check the accuracy of the data obtained by varying the collision energy represented by the solid line.

here. The agreement is good in general, somewhat better for $v^{\prime}=1$.

Figure 2 depicts the energy dependence of the reaction probability $P_{v^{\prime}-1,0}^{J=0}(E)$ for $\mathrm{D}+\mathrm{H}_{2}(v=1, j=0)$ for total angular momentum, $J=0$, and also resolved into the final vibrational states $v^{\prime}$ of the HD molecule. This calculation shows a marked qualitative difference between the results of quantum ${ }^{29}$ and classical mechanics. The smooth classical curves are in clear contrast to the pronounced resonance structure observed in the QM calculations, especially at $E_{\text {tot }}=1.20$ for $v^{\prime}=0,1$ and at 1.30 for $v^{\prime}=0,2$.

The similitudes and differences in the behavior of QCT and QM cross sections and reaction probabilities just commented on are of the same kind as the ones described for the $\mathrm{D}+\mathrm{H}_{2}(v=0, j=0)$ case. $^{46}$

A representation of the variation of the integral cross sections for $\mathrm{D}+\mathrm{H}_{2}(v=1, j) \rightarrow \mathrm{HD}\left(v^{\prime}\right)+\mathbf{H}$ with the initial rotational number $j$ of the $\mathrm{H}_{2}$ molecule at several values of the collision energy is given in Fig. 3. Similar calculations, albeit without resolution into the final vibrational states of the product molecule, had been first carried out for $\mathrm{H}+\mathrm{H}_{2}(v=0)$ by Boonenberg and Mayne ${ }^{48}$ and later for $\mathrm{D}+\mathrm{H}_{2}(v=0,1,2)$ by Sathyamurthy and Toennies ${ }^{34}$ and by Aoiz et al. ${ }^{35,36}$ These calculations showed that an increase in the rotational quantum number $j$ of $\mathrm{H}_{2}$ causes the cross section to go through a minimum at about $j=4-6$ for collision energies below $E_{T}=0.65 \mathrm{eV}$ and for the three values of the initial $v$ number studied. The resolution into the three vibrational exit channels for $\mathrm{D}+\mathrm{H}_{2}(v=1)$ leads to different types of behavior in $\sigma_{R}(j)$. The reaction cross section as a function of $j$ shows for the various $E_{T}$ values clear minima at $j=6-8$ for $v^{\prime}=0$, less pronounced minima to a smooth decline for $v^{\prime}=1$, and marked monotonic increases for $v^{\prime}=2$. These minima are commonly attributed to an "orientation effect" particularly important at low energies, where the steric hindrances of the PES have a

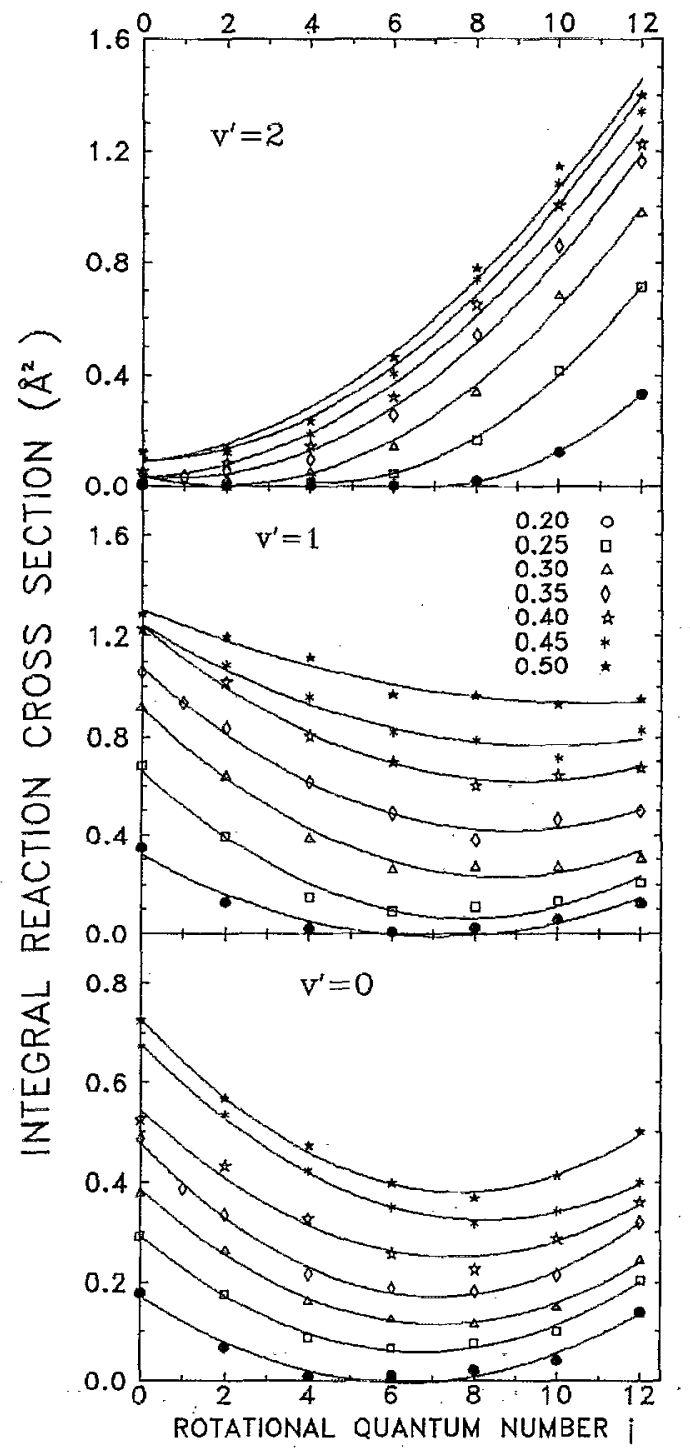

FIG. 3. Integral reaction cross section $\left(\AA^{2}\right)$ into the HD final vibrational states $v^{\prime}=0,1,2$ as a function of the initial rotational quantum state of the $\mathrm{H}_{2}$ molecule at the indicated collision energies. Lines through the points are least-squares fits to the calculated values.

greater influence. Taking that into account one can expect the behavior of $\sigma_{R}(j)$ portrayed in Fig. 3. An increase in the translational energy of the reagents tends to flatten the minima present for $v^{\prime}=0$ and 1 . The amount of energy needed for the opening of the $v^{\prime}=2$ channel is high enough to reduce the relative contribution of the orientation effect and therefore the total cross section grows monotonically with increasing rotational energy of the $\mathrm{H}_{2}$ molecule. Comparable values of $\sigma_{R}(j)$ extending to high $j$ numbers are not available from accurate $\mathrm{QM}$ calculations.

An extensive collection of the partial integral cross sections for $\mathrm{D}+\mathrm{H}_{2}(v=1, j=0) \rightarrow \mathrm{HD}\left(v^{\prime}, j^{\prime}\right)+\mathrm{H}$ resolved in both the vibrational and rotational states of the $\mathrm{HD}$ product is given in Table S-1 of Ref. 49. A comparison of these cross sections with the accurate QM calculations of Zhang and Miller (Table V of Ref. 29) shows overall good accordance. At collision energies below $0.4 \mathrm{eV}$ the absolute 

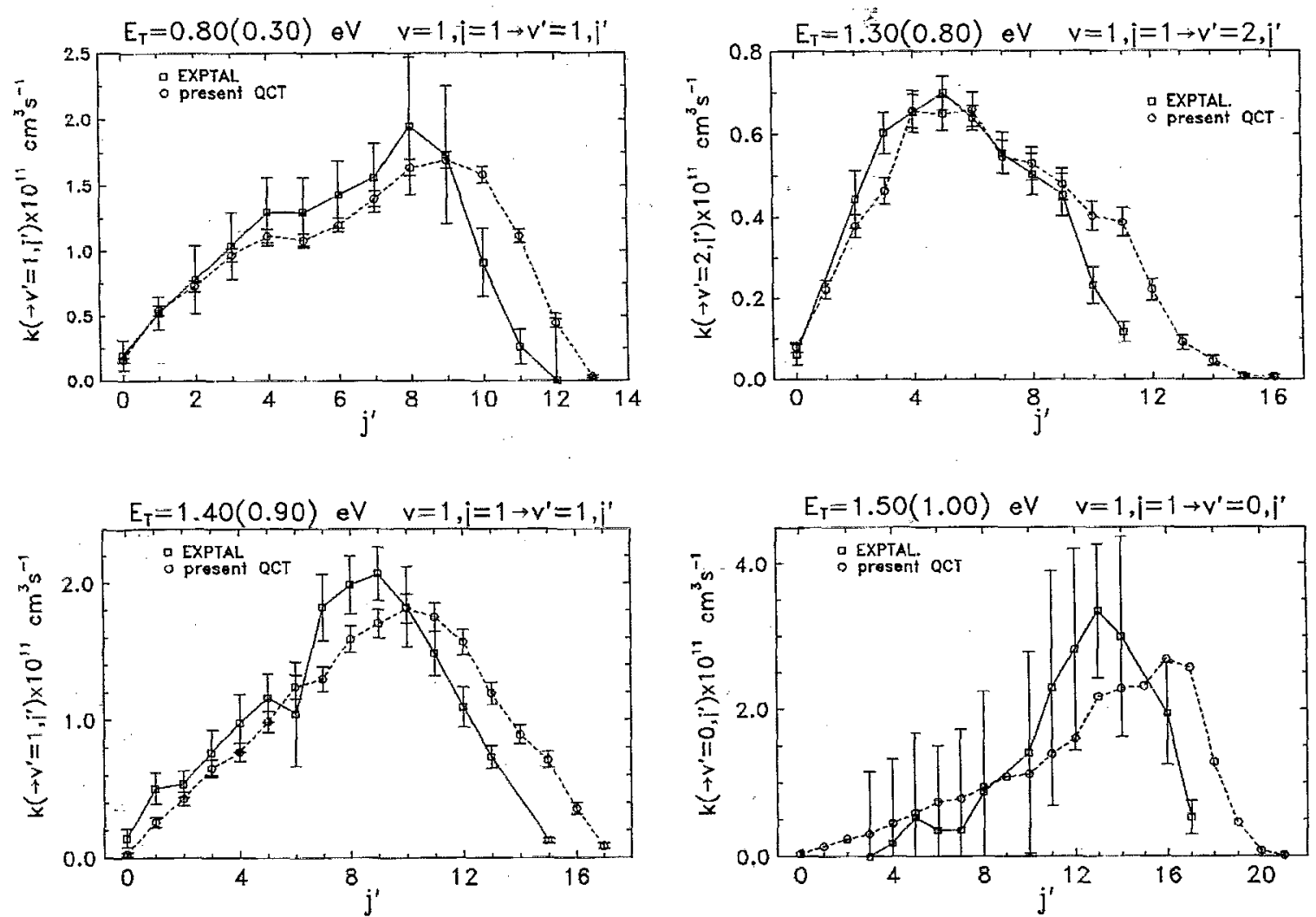

FIG. 4. Final state resolved rate constants for the $\mathrm{D}+\mathrm{H}_{2}(v=1, j=1) \rightarrow \mathrm{HD}\left(v^{\prime}, j^{\prime}\right)+\mathrm{H}$ reaction in units of $\mathrm{cm}^{3} \mathrm{~s}^{-1}$. Solid line-squares are the experimental results of Ref. 39, the dashed line and circles are present QCT calculations. The experimental points have been scaled to the QCT rate constants by least squares taking into account the uncertainties of both sets of data. The energies of the fast and slow (in parentheses) channels are indicated in each case. For more details see Sec. II.

values and the shape of the rotational distributions are coincident. At higher energies the classical $j^{\prime}$ distributions for both $v^{\prime}=0,1$ are hotter than the QM ones. In addition and as already mentioned, the absolute values of the $\mathrm{QM}$ cross sections are systematically higher for the $v^{\prime}=1$ channel at energies above $0.40 \mathrm{eV}$.

Figure 4 shows a comparison between the present QCT and the latest state specific relative rate constants determined by the group of Zare $\mathrm{Z}^{39,40}$ by using the experimental laser techniques mentioned in Sec. I. In all the cases shown, DI was used as the photolytic precursor of the D atoms. Previous measurements at $E_{T} \simeq 1 \mathrm{eV}$ using $\mathrm{DBr}$ had led to problems in the detection of $\operatorname{HD}\left(v^{\prime}=1, j^{\prime} \geqslant 8\right)$ attributable most probably to space-charge effects. ${ }^{39,40}$ As already discussed, a noteworthy discrepancy between the experimental and the theoretical (from both $\mathrm{QCT}^{41,43}$ and $\mathrm{QM}^{41-43}$ calculations) distribution of rotational states of the $\operatorname{HD}\left(v^{\prime}=1\right)$ product molecule was found precisely for $j^{\prime} \geqslant 8$. Nevertheless, recent $\mathrm{QM}$ calculations by $\mathrm{Wu}$ and Kuppermann, ${ }^{44,45}$ that take into account the effect of the geometric phase induced by the conical intersection between the two lowest potential energy surfaces of $\mathrm{H}_{3}$, show good concordance with this experiment.

Of all the DI experimental results appearing in Fig. 4, the one at $E_{T}=0.8 \mathrm{eV}$ corresponds to an independentlaser-photolysis (ILP) experimental geometry ${ }^{39}$ whereas those represented in the other three panels have been ob- tained with a probe-laser-photolysis (PLP) arrangement. ${ }^{39}$ In the ILP geometry, the $v^{\prime}, j^{\prime}$ state of the HD molecule detected does not depend on the collision energy. In the PLP setup where the probe and photolysis lasers are the same, the rovibrational state detected is related to the collision energy. The relative amount of $I^{*}\left({ }^{2} P_{1 / 2}\right)$ and I $\left({ }^{2} P_{3 / 2}\right)$ and, consequently, that of slow and fast $\mathrm{D}$ atoms changes markedly over the range of photolysis wavelengths used. ${ }^{50}$ A precise theoretical simulation of the data from the PLP measurements is rendered difficult by the characteristics of these experiments. In addition to the spread in $E_{T}$, one must take into account the fact that each one of the PLP points represented in Fig. 4 corresponds to a different value of the average collision energy and of the ratio of faster to slower $D$ atoms participating in the reaction. All these details have been considered by Neuhauser et $a l^{40}$ in the comparison between their QM timedependent wave packet calculations and the results from the experiment. The agreement obtained is very good in general, with slight differences for the higher $E_{T}$ values. An even better agreement has been obtained by Wu and Kuppermann ${ }^{44,45}$ by including the effect of the geometric phase commented on above. The explicit consideration of this effect seems necessary in order to obtain quantitative agreement between theory and experiment for values of the total energy above $1.8 \mathrm{eV}$.

The QCT simulations represented in Fig. 4 include the 
relative contributions of the faster $\mathrm{I}\left(P_{3 / 2}\right)$ and slower $I\left(P_{1 / 2}\right)$ translational channels as described in Sec. II. Finer details as the width of the collision energy distribution (which is reported to be $0.07 \mathrm{eV} \mathrm{FWHM}{ }^{38,39}$ ) and the variation of $E_{T}$ on the rotational state detected for a given $v^{\prime}$ of $\mathrm{HD}$ [which can amount to $0.16 \mathrm{eV}$ for $v^{\prime}=0$ (Ref. 39)] have not been considered in the present simulations. The influence of the geometric phase is also not regarded in our classical calculations. The different panels of Fig. 4 are labeled after the collision energies of the faster channel. The energy of the corresponding slower channels are indicated in parentheses.

There is a good concordance between the shape of the QCT rotational distributions and those from the REMPI measurements ${ }^{39}$ and from QM calculations. ${ }^{29,40-45}$ However, the classical distributions are slightly hotter by one or two quanta. This systematic shift of the QCT rotational distributions ${ }^{51,52}$ towards higher values of $j^{\prime}$ as compared to QM calculations for $j=0$ (Ref. 29) and for $j=0,1$ (Ref. 53) and experiment ${ }^{54,55}$ was also observed for $\mathrm{D}+\mathrm{H}_{2}(v=0)$.

It has to be pointed out that the absolute values of the specific $j^{\prime}$ state resolved rate constants from Kuppermann and $\mathrm{Wu}^{45}$ are markedly larger than those from QCT and previous QM calculations. ${ }^{41-43}$ Figure 2 of Ref. 45 displays the $\mathrm{QM} j^{\prime}$ resolved rate constants for the reaction with initial $j=1$ at $E_{T}=1.00 \mathrm{eV}$ both with (GP) and without (NGP) taking into account the geometric phase. Although the two distributions are distinct, their respective peak rate constants have nearly the same value. The NGP distribution peaks at $j^{\prime}=10$ and is very similar in shape to the ones previously calculated, ${ }^{41-43}$ but while in the first case the peak rate constant is $k\left(\rightarrow j^{\prime}=10\right) \approx 4.4 \times 10^{-11} \mathrm{~cm}^{3} \mathrm{~s}^{-1}$, the one reported by Keogh et al. (from Table 3 of Ref. 43) on the Boothroyd-Keogh-Martin-Peterson (BKMP) PES is $k\left(\rightarrow j^{\prime}=10\right) \approx 3 \times 10^{-11} \mathrm{~cm}^{3} \mathrm{~s}^{-1}$. The present QCT (on the LSTH PES) value for the peak is $k\left(\rightarrow j^{\prime}=10\right)$ $=2.33 \times 10^{-11} \mathrm{~cm}^{3} \mathrm{~s}^{-1}$. The whole $j^{\prime}$ distribution, not shown here, is very similar to the one of Keogh et al. ${ }^{43}$.

It is also interesting to note the high degree of adiabaticity with respect to the interconversion of translational and vibrational energy already found in previous theoretical calculations. ${ }^{29,36}$ In fact, present results show that the average vibrational energy only changes from $\left\langle E_{v}\right\rangle=0.54$ to $0.58 \mathrm{eV}$ while the total (collision) energy changes from $1.60(0.80)$ to $2.30(1.50) \mathrm{eV}$. This seems to be confirmed by the REMPI experiment commented on above.

Figure 5 and Table S-2 (Ref. 49) show the evolution of the differential cross section for the reaction $\mathrm{D}+\mathrm{H}_{2}(v=1$, $j=0) \rightarrow \mathrm{HD}\left(v^{\prime}=0,1\right)+\mathrm{H}$ with the collision energy $E_{T}$ from 0.14 to $1.00 \mathrm{eV}$. At the lower translational energies considered, the HD molecules are scattered predominantly into the backward hemisphere with respect to the incoming $\mathrm{D}$ atom. The corresponding center of mass (c.m.) angular distributions peak at $180^{\circ}$. With increasing collision energy, these distributions become more sideways and for $E_{T}$ values beyond $\sim 0.55 \mathrm{eV}$ a distinct forward peak appears in the DCS. A qualitatively similar behavior (increase of the sideways character of the DCS with growing $E_{T}$ ) was
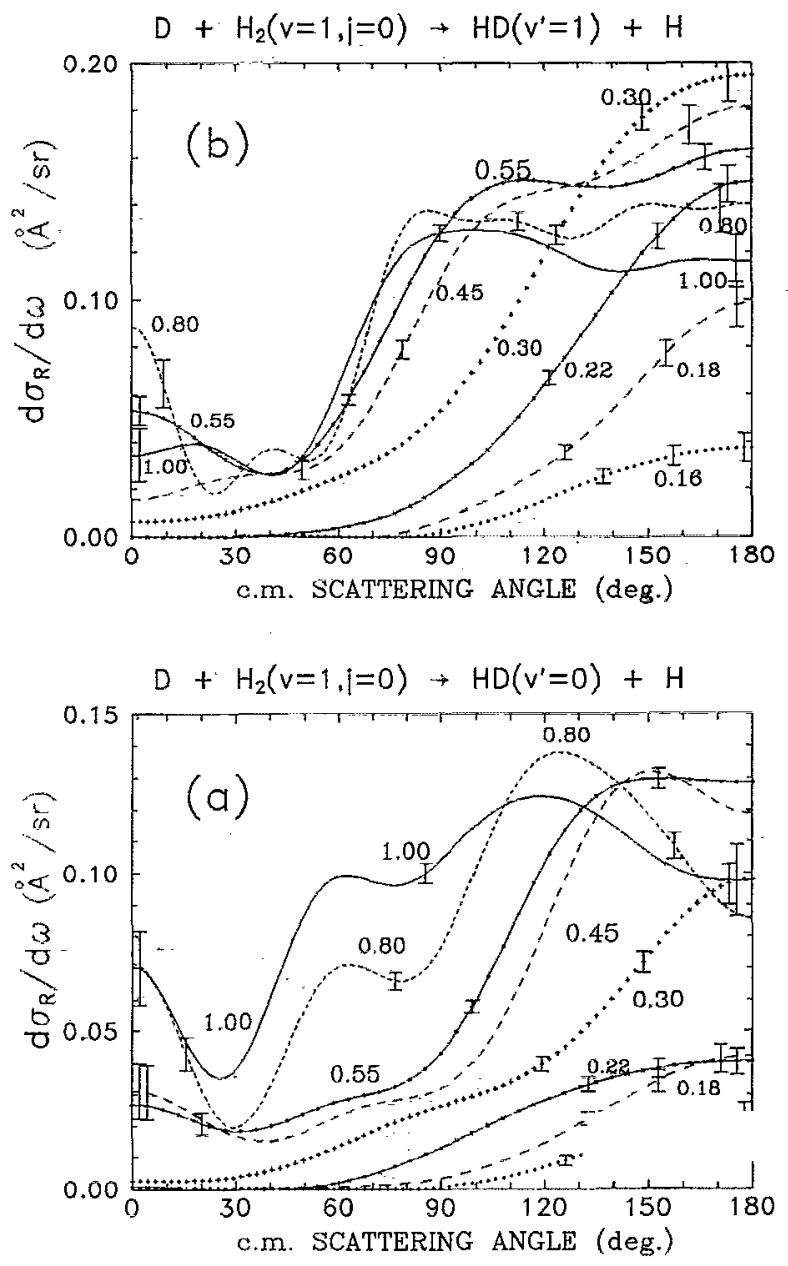

FIG. 5. Solid angle differential cross sections into $v^{\prime}=0$ (a) and $v^{\prime}=1$ (b) summed on final $j^{\prime}$ states for the $\mathrm{D}+\mathrm{H}_{2}(v=1, j=0)$ exchange reaction at the indicated collision energies $\mathrm{eV}$. Error bars represent one standard deviation.

found in the QCT calculations for $\mathrm{D}+\mathrm{H}_{2}(v=0$, $j=0) \rightarrow \mathrm{HD}\left(v^{\prime}=0,1\right)+\mathrm{H}^{46}$ However, in this case the forward scattering found for the highest collision energies does not give rise to a clear peak. The curves of Fig. 5 and data of Table S-2 corresponding to $E_{T}$ values lower than $0.55 \mathrm{eV}$ can be compared to the accurate quantummechanical results of Zhang and Miller (see Figs. 19 and 20 of Ref. 29). There is overall good agreement between QM and QCT results both in the shape and in the absolute value of the differential cross sections. In particular the marked forward peak found in the QM differential cross section for the production of $\operatorname{HD}\left(v^{\prime}=1\right)$ at $E_{T}=0.564$ is also present in the QCT results at $E_{T}=0.55 \mathrm{eV}$. The quantum-mechanical DCS for $\mathrm{D}+\mathrm{H}_{2}(v=1$, $j=0) \rightarrow \mathrm{HD}\left(v^{\prime}=1\right)+\mathrm{H}$ at $E_{T}=0.464$ and $0.564 \mathrm{eV}$ exhibit a sideways peak at about $120^{\circ}-140^{\circ}$ which is absent in the classical calculations. These QCT differential cross sections resolved in $v^{\prime}$, together with similar results for higher $j$ have been used for the simulation of the experimental angle-velocity contour plots reported in Ref. 33 for $\bar{E}_{T}$ $=0.24,0.28$, and $0.30 \mathrm{eV}$. The agreement between the measurements and the calculations is very good. The maxi- 

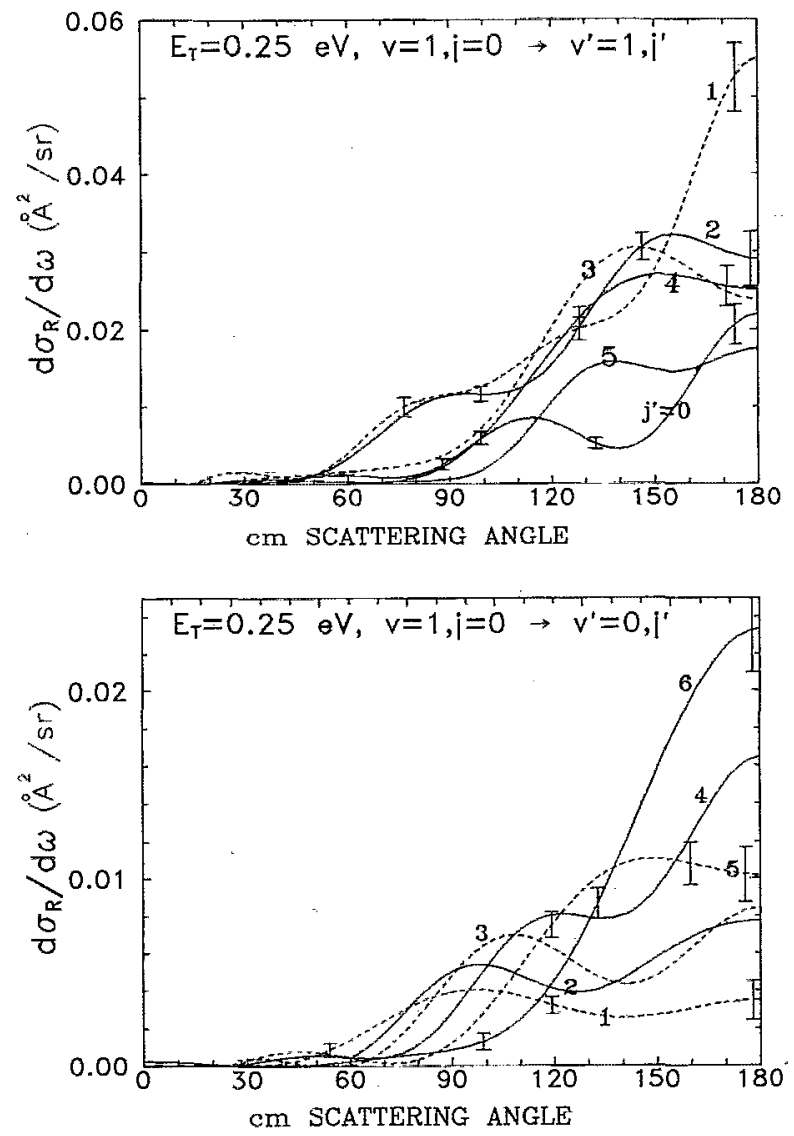

FIG. 6. Solid angle differential cross sections for the $\mathrm{D}+\mathrm{H}_{2}(v=1$, $j=0) \rightarrow \mathrm{HD}\left(v^{\prime}, j^{\prime}\right)+\mathrm{H}$ reaction at the collision energy of $0.25 \mathrm{eV}$. Error bars represent one standard deviation. Each curve is labeled with the corresponding $j^{\prime}$ value.

mum and the overall shape of the experimental distributions are well reproduced in all cases. Given the experimental resolution, no separation of the contributions from the final vibrational states is observed.

The QCT differential cross sections for $\mathrm{D}+\mathrm{H}_{2}(v=1$, $j=0) \rightarrow \mathrm{HD}\left(v^{\prime}\right)+\mathrm{H}$ become more sideways and their maxima shift from $180^{\circ}$ to lower angles with increasing collision energy. The forward peaks get also more prominent up to $0.80 \mathrm{eV}$ and then they become less pronounced; this is particularly noteworthy for $v^{\prime}=1$, as can be seen in Fig. 5(b). The summed over $j^{\prime}$ DCS for $\mathbf{D}+\mathrm{H}_{2}(v=1$, $j=1) \rightarrow \mathrm{HD}\left(v^{\prime}=0,1\right)+\mathrm{H}$ at $E_{T}=1.0 \mathrm{eV}$ tabulated in Table S-3 (Ref. 49) are similar to the one for $j=0$ and can be compared to other theoretical calculations. These calculations include accurate $\mathrm{QM}$ results on the $\mathrm{DMBE}^{41-43}$ and BKMP $^{43}$ surfaces as well as QCT on the DMBE PES. ${ }^{41,43}$ The differential cross section obtained here on the LSTH surface for $v^{\prime}=1$ is very similar to the one on the DMBE for $j=1$ in shape and absolute value, sharply peaking at $90^{\circ}$ and with a forward peak somewhat less pronounced than those given by QM calculations and with a slightly smaller backward contribution.

Figure 6 shows the differential cross section for $\mathrm{D}+\mathrm{H}_{2}(v=1, j=0) \rightarrow \mathrm{HD}\left(v^{\prime}=0,1 ; j^{\prime}\right)+\mathrm{H}$ resolved in $j^{\prime}$
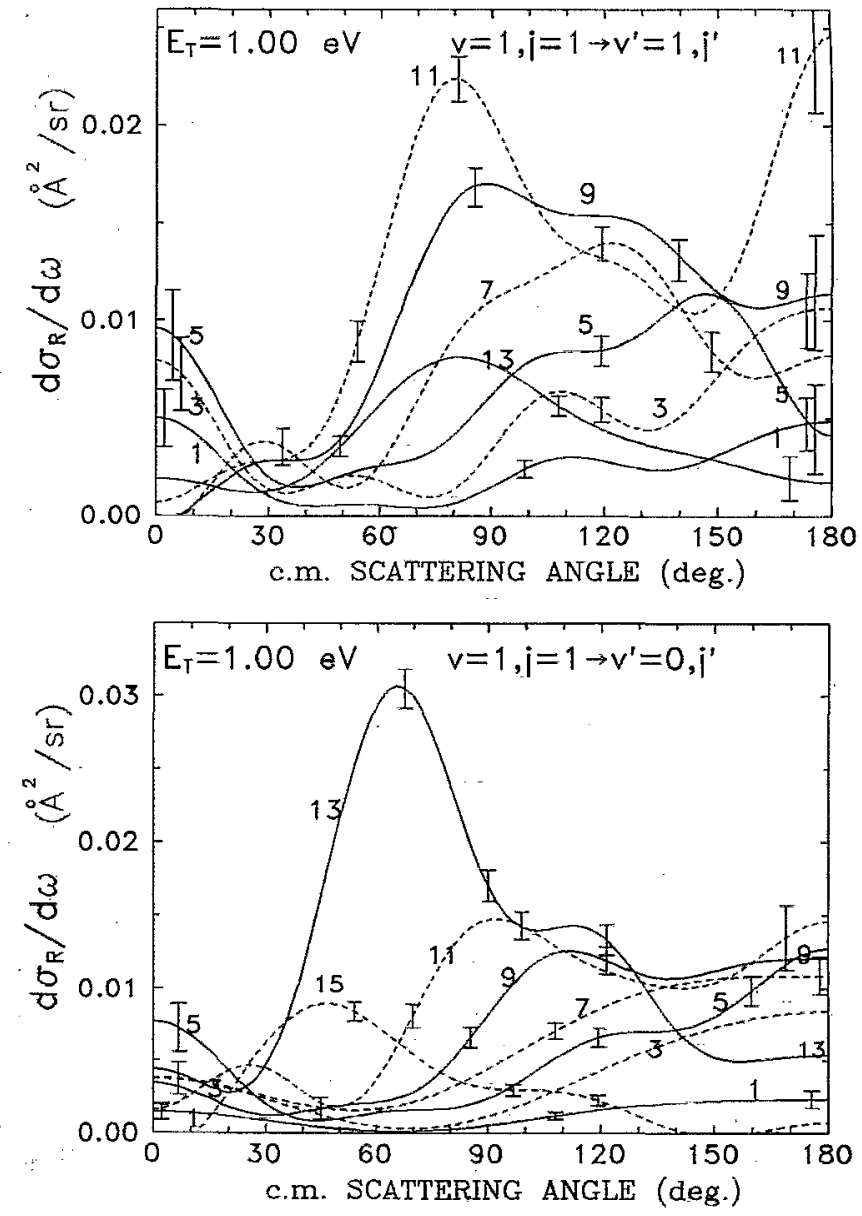

FIG. 7. Same as Fig. 6 but for $\mathrm{D}+\mathrm{H}_{2}(v=1, j=1) \rightarrow \mathrm{HD}\left(v^{\prime}, j^{\prime}\right)+\mathrm{H}$ and $E_{T}=1.00 \mathrm{eV}$. For clarity only DCS corresponding to odd $j^{\prime}$ are shown.

at $E_{T}=0.25 \mathrm{eV}$. Additionally, Table S-4 of Ref. 49 contains similar results for $E_{T}=0.55$ and $0.80 \mathrm{eV}$. Similarly, Fig. 7 displays the $v^{\prime}, j^{\prime}$ resolved differential cross section for the $\mathrm{D}+\mathrm{H}_{2}(v=1, j=1)$ reaction at $E_{T}=1.00 \mathrm{eV}$. The lower panels of these figures are the results for $v^{\prime}=0$ and the upper ones for $v^{\prime}=1$. At the lowest collision energy shown $\left(E_{T}=0.25 \mathrm{eV}\right)$ the scattering of HD is mostly backward for all the $j^{\prime}$ values and for both $v^{\prime}$ states. The sideways character of the DCS increases with collision energy. For $E_{T}=0.55$ and $0.80 j=0$ and for $1.00 \mathrm{eV} j=1$ the DCS for the lowest $j^{\prime}$ have, in general, maxima close to $180^{\circ}$ but these maxima shift towards lower angles with growing $j^{\prime}$. It is interesting to note that the forward contributions to the differential cross sections of Fig. 5 are due to the lowest $j^{\prime}$ values. A similar trend was found in QCT and in accurate $\mathrm{QM}$ calculations for the $\mathrm{D}+\mathrm{H}_{2}(v=0, j=0)$ reaction..$^{46,53,56,57}$ In that case the most forward contributions to the low differential cross sections were related to longer lived trajectories. ${ }^{46}$

The $j^{\prime}$ state resolved differential cross sections for $E_{T}$ $=1.0 \mathrm{eV} v=j=1 \rightarrow v^{\prime}=1$ (upper panel of Fig. 7) can be directly compared to the ones recently reported by Kuppermann and $\mathrm{Wu}^{45}$ for $j^{\prime}$ values from 5 to 10 calculated without and with the geometrical phase effect. Both sets of 

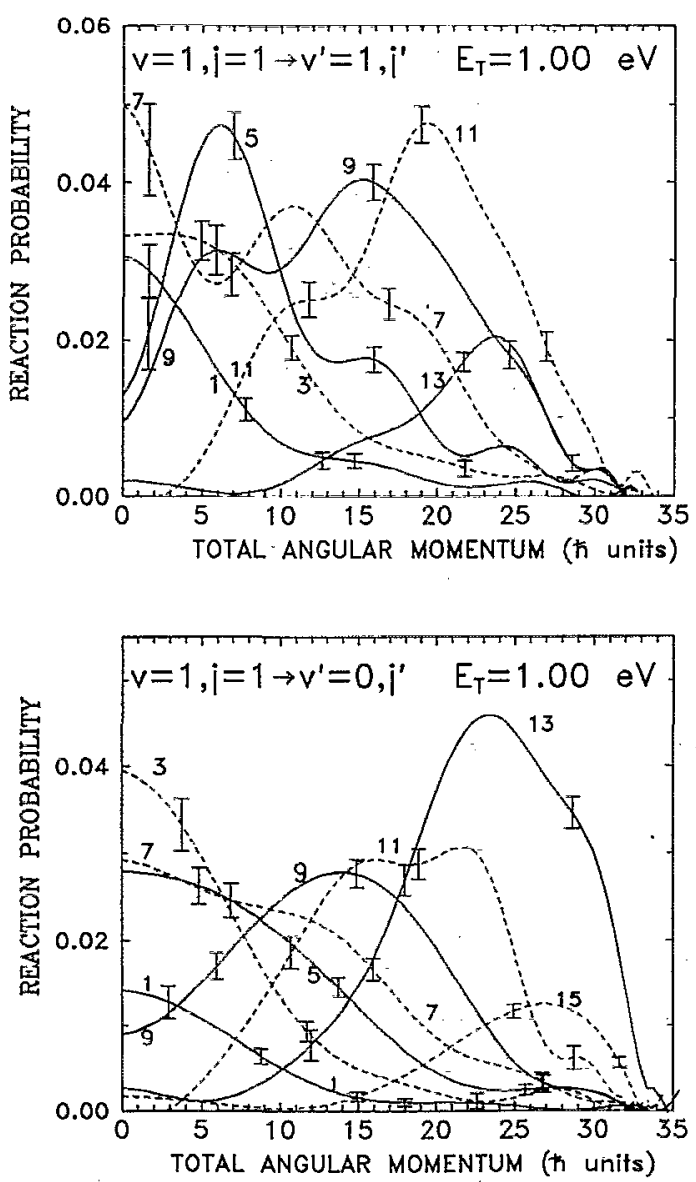

FIG. 8. Reaction probability as a function of the total angular momentum for the $\mathrm{D}+\mathrm{H}_{2}(v=1, j=1) \rightarrow \mathrm{HD}\left(v^{\prime}, j^{\prime}\right)+\mathrm{H}$ reaction at the collision energy of $1.00 \mathrm{eV}$. Calculation uncertainties (one standard deviation) are represented by error bars.

differential cross sections are similar in the tendency to sideways scattering and in the peak shifting towards lower angles as $j^{\prime}$ increases. However, there are noticeable differences. Most of the quantal DCS, particularly those obtained by taking into account the geometric phase effect, have two maxima, whereas the classical DCS show two peaks only for $j^{\prime}>9$. For $j^{\prime}=5$ the QM DCS is sideways with negligible forward scattering, whereas the QCT one is more backward with a forward peak. Overall, as it could be expected, the agreement is better for the DCS calculated without the geometrical phase effect and the absolute values are very similar. ${ }^{45(b)}$ The DCS calculated with the geometrical phase effect, as in the case of the integral cross sections, have sensibly higher values for low $j^{\prime}$ and smaller for high $j^{\prime}$.

Figure 8 represents the $j^{\prime}$ resolved reaction probabilities as a function of the total angular momentum, $P(J)$ for $E_{T}=1.0 \mathrm{eV}$ and $j=1$. Table S-5 (Ref. 49) includes the $P(J)$ for $E_{T}=0.25,0.55$, and $0.80 \mathrm{eV}$ for initial $j=0$. In general, the higher the $j^{\prime}$ state the larger are the $J$ values implied in the scattering; note however that for the lowest $j^{\prime}$ there is a tail in $P(J)$ that extends to the highest $J$ values accessible at each energy. A comparison of these reaction probabilities with the corresponding DCS of Figs. 6 and 7 and Table S-4 shows the correlation between the scattering angle and $J$. In fact, the forward features in the differential cross sections are attributable to the just mentioned tails in the $P(J)$ functions for the smallest $j^{\prime}$. The $P(J)$ tend to peak at increasingly higher $J$ values as $j^{\prime}$ increases. Some of the distributions are bimodal, this is especially the case at $1.0 \mathrm{eV} j=1$ (Fig. 8) and $j^{\prime}$ values higher than 5 in accordance with the already shown DCS. The partial state to state cross section $\sigma_{v^{\prime} j^{\prime}}^{\mathrm{J}}$, can be deduced from the $P(J)$ by

$$
\sigma_{v^{\prime} j^{\prime}}^{\mathrm{J}}=\frac{\pi \hbar^{2}}{2 \mu E_{T}} \int_{\mathrm{J}-1 / 2}^{\mathrm{J}+1 / 2}(2 J+1) P_{v^{\prime} j^{\prime}}(J) d J .
$$

For $v^{\prime}=1 j^{\prime}=7-10$ the $\sigma_{v^{\prime} j^{\prime}}^{\mathrm{J}}$ obtained from the $P(J)$ of Fig. 8 can be compared with the QM results of Keogh et al. ${ }^{43}$ The agreement between both sets of $\sigma_{v^{\prime} j^{\prime}}^{\mathrm{J}}$ distributions is fairly good and QCT can reproduce most of the features of the $\mathrm{QM}$ calculations.

A three-dimensional representation of the differential cross section summed on $j^{\prime}$ for $\mathrm{D}+\mathrm{H}_{2}(v=1, j=0)$ $\rightarrow \mathrm{HD}\left(v^{\prime}=0,1\right)+\mathrm{H}$ is given in Fig. 9 as a function of the c.m. scattering angle and of total energy. For the two vibrational exit channels considered, the backward peak in the DCS is an oscillating function of energy. Oscillations in the backward scattering were reported for the ground-state reaction in accurate $\mathrm{QM}$ calculations by Zhang and Miller $^{29}$ and corroborated by Kornweitz, Persky, and Baer $^{58-60}$ for $\mathrm{D}+\mathrm{H}_{2}(v=0)$ and for $\mathrm{D}+\mathrm{H}_{2}(v=1)$ in highly detailed QCT calculations. The dynamical interpretation of these oscillations is not yet clear and deserves a thorough study. The forward structures apparent in Fig. 9 for both $v^{\prime}$ states at total energies higher than about $1.1 \mathrm{eV}$ are mainly due to scattering of HD molecules with low rotational quantum numbers as already discussed (see Figs. 5-7 and Tables S-2 to S-4). A qualitative similar behavior was found in the $\mathrm{D}+\mathrm{H}_{2}(v=0)$ reaction ${ }^{29,46}$ [see Figs. 9(e) and 9(f) of Ref. 46]. In the present case, however, higher total energies are accessed and the forward structures are more pronounced. Figure 10 shows that forward scattering for $\mathrm{D}+\mathrm{H}_{2}(v=1)$ also corresponds to trajectories with longer collision times $\tau_{\text {col }}$, where $\tau_{\text {col }}$ is defined, according to Ref. 46, as the time during which the radial energy is lower than the potential energy.

\section{CONCLUSIONS}

Extensive QCT calculations on the dynamics of the $\mathrm{D}+\mathrm{H}_{2}(v=1) \rightarrow \mathrm{HD}+\mathrm{H}$ reaction have been performed in order to compare with the different experimental data and with the results from accurate quantum-mechanical calculations that have become available over the last years.

The agreement between the present thermal rate constants resolved into the final states of the products and those from the latest experiments ${ }^{1}$ is very good. A similar good agreement is obtained with the experimental absolute value of the total cross section. ${ }^{31}$ The calculated differential cross sections can satisfactorily reproduce the anglevelocity contour plots obtained in a crossed molecular beam experiment. ${ }^{33}$ The accord between the QCT state specific rate constants and those from REMPI experiments 

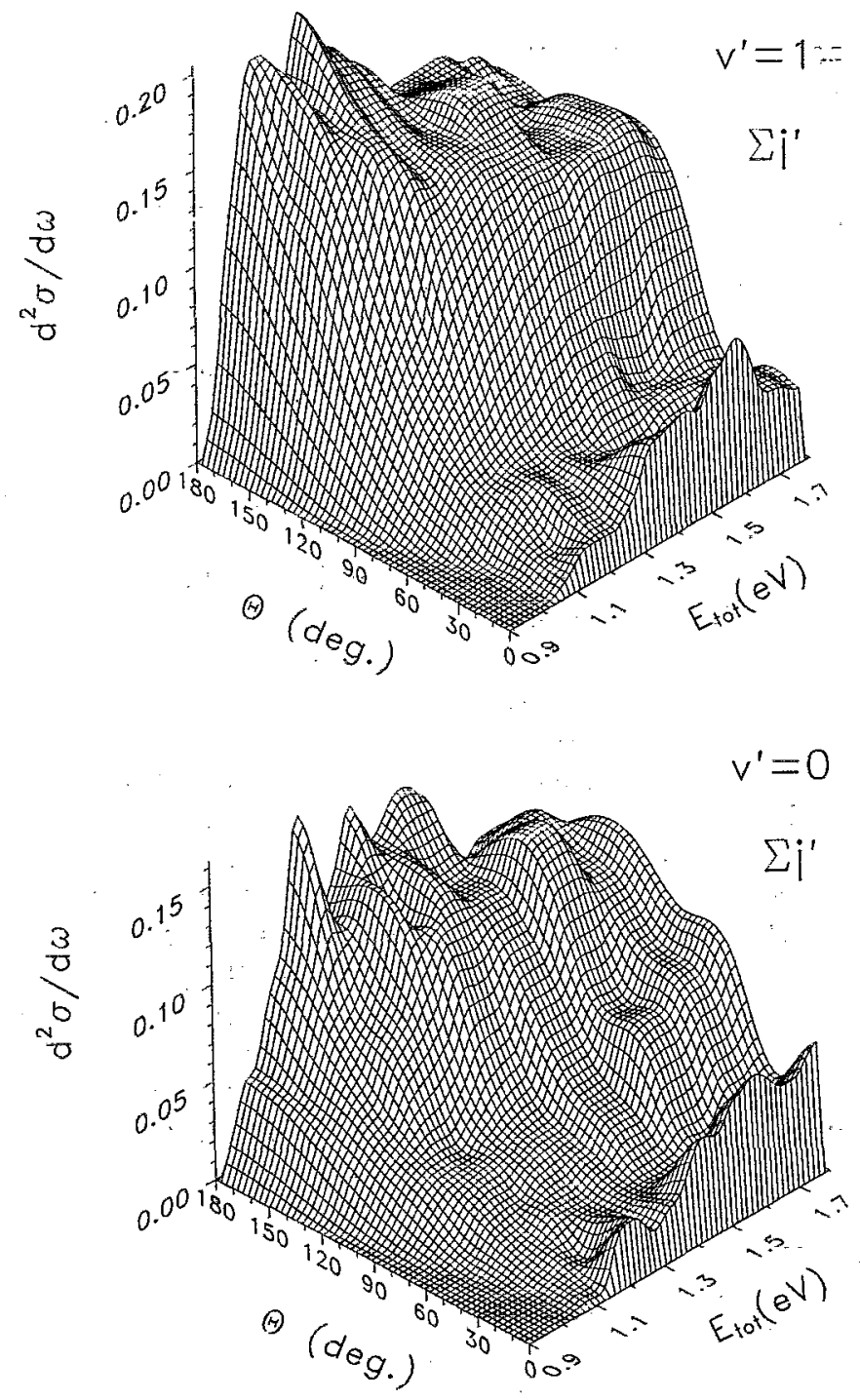

FIG. 9. Three-dimensional plots of the differential cross sections (in $\AA^{2} \mathrm{sr}^{-1}$ ) as a function of the scattering angle and total energy for the $\mathrm{D}+\mathrm{H}_{2}(v=1, j=0)$ into $v^{\prime}=0$ (bottom) and $v^{\prime}=1$ (top) summed on final $j^{\prime}$ states.

is reasonable, although the calculated rotational distributions tend to be hotter than the measured ones.

The comparison of QM and QCT translational excitation functions resolved in final vibrational states reveals a very good coincidence, mainly for collision energies below $0.4 \mathrm{eV}$. Similarly, the rate constants from $v=1, j=0$ given by QM and QCT calculations are in overall good accordance, even at low temperatures. However, the QM evolution of the reaction probabilities with the total energy for zero total angular momentum shows resonance structures that are absent in the classical calculations. The rotational distributions from $\mathrm{QM}$ calculations $\mathrm{s}^{29,40-45}$ are cooler by one or two quanta than the present ones, but in some cases, slightly hotter than the measured ones. A recent study by Wu and Kuppermann ${ }^{44,45}$ indicates that for energies higher than $1.8 \mathrm{eV}$ the effect of the geometric phase cannot be neglected in order to get quantitative agreement with ex-

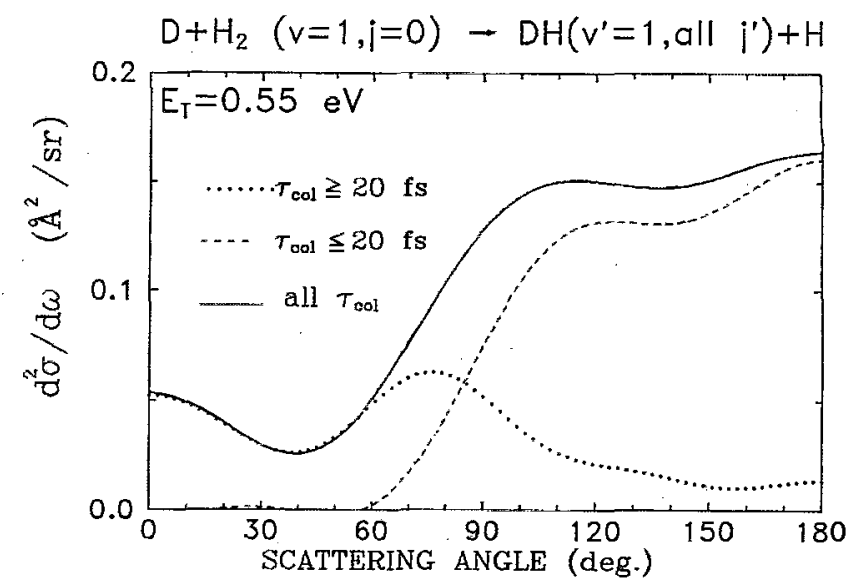

FIG. 10. Solid angle differential cross sections at $E_{T}=0.55 \mathrm{eV}, v=1$, $j=0 \rightarrow v^{\prime}=1$, summed on $j^{\prime}$. The contributions of trajectories with short (dashed line) and with long (dotted line) collision times are separated.

periment. This effect is not included in our calculations. On the other hand, the QM absolute values of the rotationally state resolved specific rate constants are, in general, larger than present ones. ${ }^{41-43,45}$ The $j^{\prime}$ state resolved differential cross sections from Ref. 45 at the collision energy of 1.00 $\mathrm{eV}$ and initial $j=1$, especially the ones that take into account the geometric phase, show some differences in shape as compared with present $\mathrm{QCT}$ results.

The evolution of the QCT differential cross sections for $\mathrm{D}+\mathrm{H}_{2}(v=1)$ with energy shows general trends similar to those already found for $\mathrm{D}+\mathrm{H}_{2}(v=0)$, i.e., the DCS resolved into the final vibrational states become broader and sideways peaked with increasing $E_{T}$ and eventually gives rise to a forward peak in general agreement with the ones obtained in QM calculations. Resolving in final rotational states $j^{\prime}$ the DCS become more sideways with increasing $j^{\prime}$, However, the mentioned forward peak corresponds to the lowest $j^{\prime}$ values and, interestingly, this feature is associated with the longest lived trajectories in analogy with the results of the reaction on the ground vibrational state.

\section{ACKNOWLEDGMENTS}

The authors wish to thank Professor J. Peter Toennies for his stimulation in carrying out this work. They also gratefully acknowledge the German-Spanish scientific exchange program "Acciones Integradas HispanoAlemanas," under Project No. HA-063. H. K. B. especially wishes to thank $K$. Bammel and $U$. Tappe for their assistance with present QCT calculations. F. J. A., V. J. H., and V.S. R. also acknowledge the financial support by the DGICYT under Project No. PB92-0219-C03.

${ }^{1} \mathrm{H}$. Buchenau, J. P. Toennies, J. Arnold, and J. Wolfrum, Ber. Bunsenges. Phys. Chem. 94, 1231 (1990), and references therein.

${ }^{2}$ (a) P. Siegbahn and B. Liu, J. Chem. Phys. 68, 2457 (1978); (b) D. G. Truhlar and C. J. Horowitz, ibid. 68, 2466 (1978); 71, 1514E (1979).

${ }^{3}$ A. J. C. Varandas, F. B. Brown, C. A. Mead, D. G. Truhlar, and B. C. Garrett, J. "Chem. Phys. 86, 6258 (1987).

${ }^{4}$ C. W. Bauschlicher, Jr., S. R. Langhoff, and H. Partridge, Chem. Phys. Lett. 170, 345 (1990). 
${ }^{5}$ A. I. Boothroyd, W. J. Keogh, P. G. Martin, and M. R. Peterson, J. Chem. Phys. 95, 4343 (1991).

${ }^{6}$ E. B. Gordon, B. I. Ivanov, A. P. Perminov, E. S. Medvedev, A. N. Ponomarev, and V. L. Tal'Rose, Chem. Phys. 8, 147 (1975).

${ }^{7}$ E. B. Gordon, B. I. Ivanov, A. P. Perminov, V. G. Balalaev, A. N. Ponomarev, and V. V. Filatov, Chem. Phys. Lett. 58, 425 (1978).

${ }^{8}$ M. Kneba, V. Wellhausen, and J. Wolfrum, Ber. Bunsenges. Phys. Chem. 83, 940 (1979).

${ }^{9}$ U. Wellhausen and J. Wolfrum, Ber. Bunsenges. Phys. Chem. 89, 314 (1982).

${ }^{10}$ G. P. Glass and B. K. Chaturvedi, J. Chem. Phys. 77, 3478 (1982).

${ }^{11}$ V. B. Rozenshtein, Y. M. Gershenzon, A. V. Ivanov, S. D. Il'in, and S. I. Kucheryavii, Chem. Phys. Lett. 105, 423 (1984).

${ }^{12}$ V. B. Rozenshtein, Y. M. Gershenzon, A. V. Ivanov, S. D. Il'in, S. I. Kucheryavii, and S. Y. Umanskii, Chem. Phys. Lett. 121, 89 (1985).

${ }^{13}$ U. Wellhausen and J. Wolfrum, Ber. Bunsenges. Phys. Chem. 89, 316 (1985).

${ }^{14}$ G. D. Barg, H. R. Mayne, and J. P. Toennies, J. Chem. Phys. 74, 1017 (1981).

${ }^{15}$ H. R. Mayne and J. P. Toennies, J. Chem. Phys. 75, 1794 (1981).

${ }^{16}$ J. C. Sun, B. H. Choi, R. T. Poe, and K. T. Tang, Phys. Rev. Lett. 44, 1211 (1980).

${ }^{17}$ J. M. Bowman, K. T. Lee, and R. B. Walker, J. Chem. Phys. 79, 3742 (1983).

${ }^{18}$ E. Pollak and R. E. Wyatt, J. Chem. Phys. 78, 4464 (1983).

${ }^{19}$ R. B. Walker and E. F. Hayes, J. Phys. Chem. 87, 1255 (1983).

${ }^{20}$ N. Abu Salbi, D. J. Kouri, Y. Schima, and M. Baer, Chem. Phys. Lett. 105, 472 (1984).

${ }^{21}$ N. Abu Salbi, D. J. Kouri, Y. Schima, and M. Baer, J. Chem. Phys. 82, 2650 (1985).

${ }^{22}$ B. C. Garrett and D. G. Truhlar, J. Phys. Chem. 89, 2204 (1985).

${ }^{23}$ B. C. Garrett, D. G. Truhlar, A. J. C. Varandas, and N. C. Blais, Int. J. Chem. Kinet. 18, 1065 (1986).

${ }^{24}$ R. B. Walker and E. Pollak, J. Chem. Phys. 83, 2851 (1985).

${ }^{25}$ E. Pollak, N. Abu Salbi, and D. J. Kouri, Chem. Phys. Lett. 113, 585 (1985).

${ }^{26}$ K. Haug, D. W. Schwenke, Y. Schima, D. G. Truhlar, J. Zhang, and D. J. Kouri, J. Phys. Chem. 90, 6757 (1986).

${ }^{27}$ T. Dreier and J. Wolfrum, Int. J. Chem. Kinet. 18, 919 (1986).

${ }^{28}$ J, Arnold, Ph.D. thesis, Universität Heidelberg (1990), cited in Ref. 1.

${ }^{29}$ J. Z. H. Zhang and W. H. Miller, J. Chem. Phys. 91, 1528 (1989).

${ }^{30}$ R. Götting, J. P. Toennies, and M. Vodegel, Int. J. Chem. Kinet. 18, 949 (1986).

${ }^{31}$ R. Götting, V. Herrero, J. P. Toennies, and M. Vodegel, Chem. Phys. Lett. 137, 524 (1987).

${ }^{32}$ M. Vodegel, Ph.D. thesis, Universität Göttingen (1987). Report of the Max-Planck Institut für Strömungsforschung 10/1988.

${ }^{33}$ (a) H. Buchenau, Ph.D. thesis, Universität Göttingen (1991). Report of the Max-Planck Institut für Strömungsforschung 5/1992. (b) $\mathrm{H}$. Buchenau, V. J. Herrero, J. P. Toennies, and M. Vodegel (to be published).

${ }^{34}$ N. Sathyamurthy and J. P. Toennies, Chem. Phys. Lett. 143, 323 (1988).

${ }^{35}$ F. J. Aoiz, V. J. Herrero, and V. Sáez Rábanos, Chem. Phys. Lett. 161, 270 (1989).
${ }^{36}$ F. J. Aoiz, V. J. Herrero, and V. Sáez Rábanos, J. Chem. Phys. 94, 7991 (1991).

${ }^{37}$ D. A. V. Kliner and R. N. Zare, J. Chem. Phys. 92, 2107 (1990).

${ }^{38}$ D. A. V. Kliner, D. E. Adelman, and R. N. Zare, J. Chem. Phys. 95, 1648 (1991).

${ }^{39}$ D. E. Adelman, N. E. Shafer, D. A. V. Kliner, and R. N. Zare, J. Chem. Phys. 97, 7323 (1992).

${ }^{40}$ D. Neuhauser, R. S. Judson, D. J. Kouri, D. E. Adelman, N. E. Shafer, D. A. V. Kliner, and R. N. Zare, Science 257, 519 (1992).

${ }^{41}$ N. C. Blais, M. Zhao, D. G. Truhlar, D. W. Schwenke, and D. J. Kouri, Chem. Phys. Lett. 166, 368 (1990); 188, 368(E) (1992).

${ }^{42}$ S. L. Mielke, R. S. Friedman, D. G. Truhlar, and D. W. Schwenke, Chem. Phys. Lett. 188, 359 (1992).

${ }^{43}$ W. J. Keogh, A. I. Boothroyd, P. G. Martin, S. L. Mielke, D. G. Truhlar, and D. W. Schwenke, Chem. Phys. Lett. 195, 144 (1992).

${ }^{44}$ Y.-S. M. Wu and A. Kuppermann, Chem. Phys. Lett. 201, 178 (1993).

${ }^{45}$ (a) A. Kuppermann and Y.-S. M. Wu, Chem. Phys. Lett 205, 577 (1993); (b) the ordinate scale of Fig. 5 should be divided by 5 [A. Kuppermann and Y.-S. M. Wu, Chem. Phys. Lett. 213, 636 (1993)].

${ }^{46}$ F. J. Aoiz, V. J. Herrero, and V. Sáez Rábanos, J. Chem. Phys. 97, 7423 (1992).

${ }^{47}$ (a) W. H. Press, S. A. Teukolsky, W. T. Vetterling, and B. P. Flannery, Numerical Recipes in Fortran (Cambridge University, Cambridge, 1992); (b) P. R. Bevington, Data Reduction and Error Analysis for the Physical Sciences (McGraw-Hill, New York, 1969).

${ }^{48}$ C. A. Boonenberg and H. Mayne, Chem. Phys. Lett. 108, 67 (1984).

${ }^{49}$ See AIP document no. PAPS JCPSA-100-2789-37 for 37 pages with Tables S-1 to S-5 as supplementary material. Tables S-1 to S-4 contain integral and differential cross sections for the title reaction resolved in final states. Table S-5 shows the values of reaction probabilities as functions of the total angular momentum (see the text). Order by PAPS number and journal reference from the American Institute of Physics, Physics Auxiliary Publication Service, 500 Sunnyside Boulevard, Woodbury, New York 11797-2999. The price is $\$ 1.50$ for each microfiche (60 pages) or $\$ 5.00$ for photocopies of up to 30 pages, and $\$ 0.15$ for each additional page over 30 pages. Airmail additional. Make checks payable to the American Institute of Physics.

${ }^{50}$ I. Levy and M. Shapiro, J. Chem. Phys. 89, 2900 (1988).

${ }^{51}$ N. C. Blais and D. G. Truhlar, J. Chem. Phys. 88, 5457 (1988).

${ }^{52}$ F. J. Aoiz, V. Candela, V. J. Herrero, and V. Sáez Rábanos, Chem. Phys. Lett. 169, 243 (1990).

${ }^{53}$ M. Zhao, D. G. Truhlar, D. W. Schwenke, and D. J. Kouri, J. Phys. Chem. 94, 7074 (1990).

${ }^{54}$ D. L. Phillips, H. B. Levene, and J. J. Valentini, J. Chem. Phys. 90, 1600 (1987).

${ }^{55}$ D. A. V. Kliner, K. D. Rinnen, and R. N. Zare, Chem. Phys. Lett. 166, 107 (1990).

${ }^{56}$ R. Continetti, J. Z. H. Zhang, and W. H. Miller, J. Chem. Phys. 93, 5356 (1990).

${ }^{57}$ W. H. Miller and J. Z. Zhang, J. Phys. Chem. 95, 12 (1991).

${ }^{58}$ M. Baer, H. Kornweitz, and A. Persky, J. Chem. Phys. 92, 6635 (1990).

${ }^{59}$ H. Kornweitz, A. Persky, and M. Baer, J. Chem. Phys. 94, 5524 (1991).

${ }^{60}$ A. Persky and H. Kornweitz, Chem. Phys. Lett. 184, 479 (1991). 